\title{
Modeling of Image Formation in Multi-Spacecraft Interferometric Imaging Systems *
}

\author{
Suman Chakravorty, ${ }^{\dagger} \quad$ Pierre T. Kabamba ${ }^{\ddagger}$ and $\quad$ David C. Hyland. ${ }^{\S}$
}

\begin{abstract}
In this paper, we develop a detailed model of the process of image formation in MultiSpacecraft Interferometric Imaging Systems (MSIIS). We show that the Modulation Transfer Function of, and the noise corrupting, the synthesized optical instrument are dependent on the trajectories of the constituent spacecraft and obtain these explicit functional relationships. We show that "good" imaging using MSIIS is equivalent to painting a "large disk" with smaller "paintbrushes" while maintaining a minimum thickness of paint, given that the goal of imaging is the correct classification of the formed images. This implies that the trajectories of the constituent spacecraft have to be "dense" enough in a given region, while making sure that they are "slow" enough. This is illustrated through an example. Keywords: spacecraft formation flying, interferometry, synthetic aperture optics.
\end{abstract}

\section{Introduction}

The research presented in this paper is motivated by the prospect of taking high resolution images of extra-solar planets at distances of up to 15 parsecs. ${ }^{1}$ Other astronomical observations, such as the study of protoplanetary disks in various stages of their formation, also require high resolution imaging. This high resolution imaging is to be performed by a multi-spacecraft interferometric imaging system (MSIIS). A survey of different technologies that could be used for these missions is given in. ${ }^{2,3}$

In this paper, we model the process of image formation in an MSIIS. We also model the noise inherent in such systems. We show that both the Modulation Transfer function (MTF) of the synthesized optical instrument and the noise corrupting the image formed by such an optical instrument are dependent on the trajectories of the constituent spacecraft. Further, if we formulate the goal of imaging as the correct classification of the formed images, we show that satisfactory imaging by an MSIIS is analogous to the "painting" of a large resolution disk with smaller "coverage" disks or "paintbrushes" while maintaining a minimum thickness of paint.

The problem of design of MSIIS is related to the fields of synthetic aperture optics and formation flying. The relationship of our work to these topics is discussed next. The topic of long baseline interferometry falls under the category of synthetic aperture optics, ${ }^{4}$ that was first developed in the context of synthetic aperture radars (SAR). ${ }^{5}$ The method consists of emulating a large optical instrument by a number of smaller ones and combining their contributions in a proper way to obtain an image that has resolution comparable to that of the large optical instrument. For a discussion of the various metrics used in the optimization of these systems, please refer to ${ }^{6}$ and the references therein. All the abovementioned designs optimize the locations of the constituent telescopes such that some metric of image quality is maximized. Thus, these correspond to static optimization problems. However, for an MSIIS, due to the high resolution requirements, the "design variables" are the trajectories of the constituent spacecraft. In fact, we show the explicit dependence of the MTF on the trajectories of the constituent spacecraft. Further, we show that the noise corrupting the image in an MSIIS is a function of the spacecraft trajectories and the rate of arrival of photons on the observation plane. Given that the goal of imaging is the correct classification of images, the design of an MSIIS reduces

\footnotetext{
* This work was supported by NASA under grant NAG5-10336

$\dagger$ Post-doctoral scholar, Department of Aerospace Engineering, The University of Michigan, Ann Arbor, schakrav@engin.umich.edu

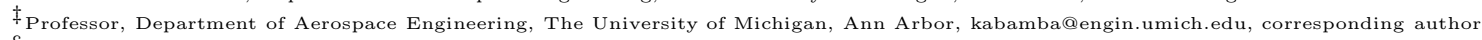

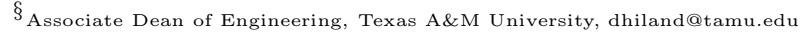


to a trajectory optimization problem, where some resource utilization of the system is minimized while satisfying the imaging constraints placed on the trajectories of the constituent spacecraft.

In recent years, there has been substantial research on the topic of multiple-spacecraft formation flying. For a detailed discussion of the issues involved, please see ${ }^{7,8}$ and the references therein. However, in the case of an MSIIS, the end goal of satellite formation flying is the synthesis of a good imaging system. With the exception of, ${ }^{9}, 10$ none of the above contributions takes the imaging aspects of the problem into account. These papers do so by coverage of the so-called $(u, v)$ plane, i.e., the spatial frequency plane, however, the adequacy of the coverage is not quantified. Moreover, the orbit design problem is not addressed: Hill orbits are used and the design problem is the optimization of the relative orientations of the constituent spacecraft. Thus, the work so far in the formation-flying literature has failed to take the specific demands of the imaging problem into consideration, which would be critical in the design of an MSIIS. In the present work, we model the image formation in an MSIIS and formulate an optimization problem that balances the imaging goals of a system with its formation flying goals. The solution to the optimization problem is addressed in a separate paper. $^{8}$

The original contributions of this paper are as follows:

- None of the work in the synthetic aperture optics literature has so far addressed the high resolution requirements inherent in the detection of extra-solar planets and other similar high-resolution astronomical observations. This work is the first attempt at modeling the "dynamics" underlying the process of image formation in such a system.

- We model the process of image formation in an MSIIS. In particular, we show that the MTF of, and the noise corrupting, the image formed by an MSIIS are dependent on the trajectories of the constituent spacecraft. Further, we show the explicit functional dependence of the above on the spacecraft trajectories.

- Given that the goal of imaging is the correct classification of an image, we show that the formation of "good" images by an MSIIS is analogous to painting a "large disk" with smaller "paintbrushes" while maintaining a minimum thickness of paint. This implies that the spacecraft relative trajectories must be "dense" enough in a given region, which is determined by the resolution specifications, while ensuring that their relative motions are "slow" enough. These requirements are explicitly quantified in this paper.

The rest of the paper is organized as follows. In section 2, we formulate the problem of design of an MSIIS. In section 3, we discuss some optics preliminaries and model the process of image formation in an MSIIS. In section 4, we model the noise inherent in such a system. In section 5, we discuss the properties of the formulated imaging model and show the analogy to "painting". In section 6 , we give a numerical example in which we illustrate the requirement that the trajectories be "dense" enough in a given region.

\section{Problem Formulation}

As mentioned in the Introduction, the design of an MSIIS should optimize some resource utilization (such as time or fuel) while taking "good" images. The design problem can then be framed as the following constrained optimization problem: "minimize the time (or fuel) required by an imaging maneuver while ensuring that the imaging constraints are satisfied." In the following, we give a mathematical formulation of the optimization problem stated above.

Let the number of spacecraft in an MSIIS be denoted by $N$. Let the trajectory of the $k^{\text {th }}$ spacecraft be denoted by $r_{k}(t) \in \Re^{3}, 1 \leq k \leq N$. Let the set of the possible trajectories be denoted by $\bar{r}$. Let $\mathcal{I}$ denote the set of images of interest and let $i \in \mathcal{I}$ be a particular image. Let $\hat{i}(\bar{r})$ be the image formed by the MSIIS following trajectory $\bar{r}$, i.e., the constituent spacecraft of the MSIIS follow the trajectories defined by $\bar{r}$. Let $Q(\hat{i}, i)$ denote an image quality metric depending on an image $i$ and its estimate $\hat{i}$. Let $T(\bar{r})$ denote the time of the imaging maneuver in following trajectory $\bar{r}$. Then, the problem of designing minimum time maneuvers for an MSIIS can be framed as follows:

$$
\min _{\bar{r}} T(\bar{r})
$$




$$
Q(i, \hat{i}(\bar{r})) \geq Q_{\text {min }}, \forall i \in \mathcal{I},
$$

where $Q_{\min }$ is a pre-specified lower bound on the performance desired of the imaging system.

Now, we can specify the image quality metric of interest to us. We note that there might be other metrics of image quality, but the following metric is consistent with the imaging application we have in mind. We assume that the images formed by the MSIIS will be used for the classification of the exo-solar planet (or any such astronomical object) into one of two classes (note that the case of more than two classes is a trivial extension of the case of two classes). The formation of an image by an optical instrument can be represented by the following convolution, ${ }^{4}$

$$
i_{p}(x, y)=h(x, y) * i(x, y)+n(x, y),
$$

where $h$ is the point spread function of the optical instrument and $n$ is the noise corrupting the image, or equivalently by the following product in the Fourier domain,

$$
I_{p}(\nu)=M(\nu) I(\nu)+N(\nu) .
$$

Here, $M$ represents the modulation transfer function (MTF) of the optical instrument. In the case of an MSIIS, the MTF function (and the psf) depend on the trajectories of the constituent spacecraft. In fact, in the next section we shall show that the noise and the MTF are both functions of the MSIIS trajectory, $\bar{x}$. Furthermore, the image is reconstructed through an operator $G$, i.e.,

$$
\hat{I}(\nu)=G(\nu) I_{p}(\nu) .
$$

In this work, for the sake of simplicity, we assume that $G(\nu)=1 / M(\nu)$. Let the set of images, $\mathcal{I}$, be partitioned into two non-empty classes, $\mathcal{I}_{1}$ and $\mathcal{I}_{2}$. We define the probability of misclassification of an estimate of some image $i \in \mathcal{I}$ as:

$$
\epsilon_{p}(\hat{i}, i)=\operatorname{prob}\left(\hat{i} \notin \mathcal{I}_{k} / i \in \mathcal{I}_{k}\right) .
$$

We say that an MSIIS (or any imaging system) performs satisfactory imaging if the following is satisfied for all $i \in \mathcal{I}$,

$$
\epsilon_{p}(\hat{i}(r), i) \leq p_{\text {min }},
$$

where $p_{\min }$ represents the maximum allowable probability of misclassification. Note that, since the MTF and the noise corrupting the image in an MSIIS are both dependent on the trajectory of the MSIIS, it follows that the estimated image and the probability of misclassification are both functions of the MSIIS trajectory. Then, we pose the design of minimum time maneuvers for an MSIIS as the following optimization,

$$
\begin{array}{r}
\min _{\bar{r}} T(\bar{r}) \\
\text { subject to } \\
\epsilon_{p}(\hat{i}(\bar{r}), i) \leq p_{\text {min }}, \forall i \in \mathcal{I} .
\end{array}
$$

We make the following observations about the optimization problem posed above:

- The optimization problem is framed as minimizing the time of the imaging maneuver subject to the "imaging constraint" that the probability of misclassification of any image in a given set be less than some pre-specified upper bound.

- We need to find the relationship between the synthesized optical instrument and the trajectories of the constituent spacecraft. 
- In general, it will be prohibitively expensive in terms of computations to evaluate the probability of misclassification given the trajectory of an MSIIS. Hence, the optimization problem posed above is not easily amenable to Mathematical Programming methods. Moreover, in general it would be prohibitively expensive to evaluate the probability of misclassification numerically, given the trajectory of the MSIIS. Coupled with the high dimensionality of the problem, this precludes the possibility of using global optimization methods such as genetic programming. The solution to the optimization problem posed above is addressed in. ${ }^{7,8}$

- Hence, we need to find sufficient conditions on the noise process corrupting the imaging system, in terms of easily evaluated second order properties of the noise process, such that the imaging constraints are satisfied. This question is addressed in. ${ }^{6}$

In the following section, we shall formulate a model for the process of image formation in an MSIIS. We shall show that the MTF of the synthesized aperture is a function of the spacecraft trajectories. Moreover, we shall show that the noise corrupting the final image formed by an MSIIS is a function of the photon arrivals on the measurement plane and the spacecraft trajectories.

\section{Equivalent Optical Aperture}

In this section, we obtain the description of the equivalent optical aperture that is created by a maneuver of an MSIIS. The propagation of an electromagnetic field from the image plane to the measurement plane is described by the Huygens-Fresnel Principle (HFP): ${ }^{4}$

$$
U(P)=\frac{1}{j \lambda} \int_{\Sigma_{I}} U(Q) \frac{e^{2 \pi j \frac{r}{\lambda}}}{r} \xi(\theta) d S,
$$

where $U(P)$ represents the value of the scalar field variable at point $P$ and $\lambda$ denotes the wavelength of the incident radiation. This situation is illustrated in Fig. 1 (the other variables in the equation are defined in the figure). Here, $\Sigma_{I}$ represents the image plane, $\Sigma_{o}$ represents the measurement or the observation plane, $P$ is a point on the observation plane, $Q$ denotes a point on the image plane, $r$ represents the distance between these points, $\theta$ and $\phi$ are the angles between the relative position vector between the points $P$ and $Q$ and the normals to the surfaces $\Sigma_{o}$ and $\Sigma_{i}$ at $P$ and $Q$ respectively, and $\xi($.$) is a known shape function. The$ above equation allows us to find the value of the electromagnetic field at a plane downfield from the image plane.

The Inverse Huygens Fresnel Principle (IHFP) expresses the field on the image plane in terms of the field on the observation plane (this is a statement of the time reversibility of light):

$$
U(Q)=\frac{-1}{j \lambda} \int_{\Sigma_{o}} U(P) \frac{e^{2 \pi j \frac{r}{\lambda}}}{r} \xi(\phi) d S
$$

In our case, the distances involved are very large and hence, to a good approximation, we have $\xi(\theta) \simeq 1$ and $\xi(\phi) \simeq 1$. In general, the electromagnetic field at the image plane is a random quantity and thus, the field measured on the observation plane is also random. The mutual intensity between two points $P_{1}$ and $P_{2}$ is defined as:

$$
J\left(P_{1}, P_{2}\right)=<U\left(P_{1}\right) U^{*}\left(P_{2}\right)>,
$$

where $x^{*}$ denotes the complex conjugate of the random variable $x$ and $<$. $>$ denotes the expectation operator. In fact, the mutual intensity is the physical quantity that is measured by a pair of spacecraft in an MSIIS. This measurement is done by evaluating the visibility and phase of the fringe that is formed by interfering the light collected by a pair of spacecraft (see pg. $338 \mathrm{in}^{11}$ ). Thus, using the IHFP and the definition of mutual intensity, it follows that,

$$
I(Q)=\frac{1}{\lambda^{2} z^{2}} \int_{\Sigma_{o}} \int_{\Sigma_{o}} J\left(P_{1}, P_{2}\right) e^{\frac{2 \pi j}{\lambda}\left(r(Q, P 1)-r\left(Q, P_{2}\right)\right)} d S_{1} d S_{2},
$$




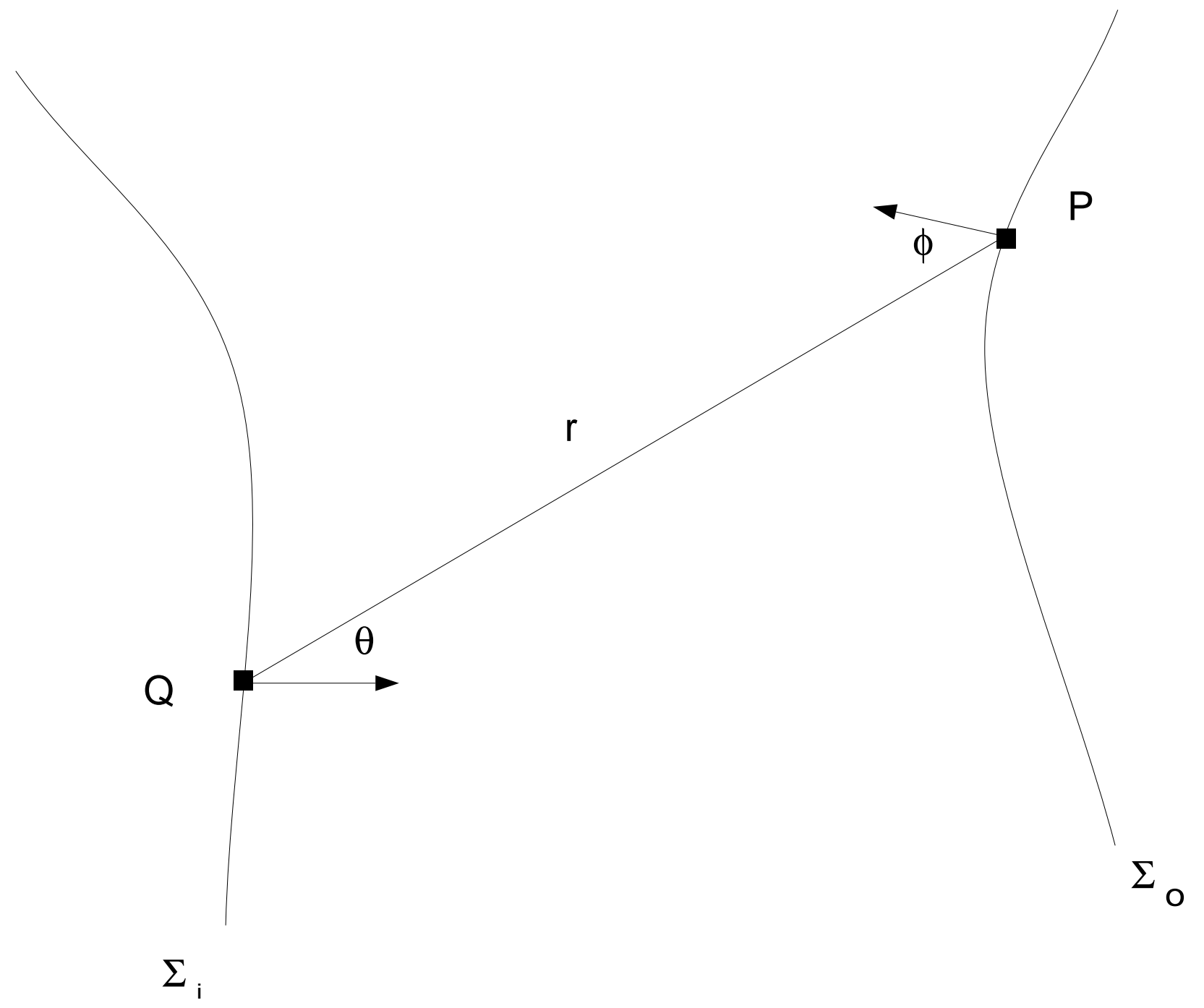

Figure 1. Propagation of Electromagnetic Field

where $I(Q)=<U(Q) U^{*}(Q)>$ denotes the intensity of the field. The situation is shown in Fig. 2. The various physical quantities of interest are shown in the figure. We assume that the electromagnetic field of the radiating object (i.e., the planet we are imaging), is incoherent. Mathematically, this means that

$$
J\left(Q_{1}, Q_{2}\right)=I\left(Q_{1}\right) \delta\left(Q_{1}-Q_{2}\right),
$$

where $\delta($.$) denotes the Dirac impulse function. Thus, the object is completely described by its intensity$ profile. Furthermore, we note from (13) that the intensity profile of the object can be evaluated by making mutual intensity measurements on the observation plane and using (13). When the mutual intensity measurements are corrupted by noise, it can be seen that (13) represents the minimum variance unbiased Gauss-Markov estimate of the object. Using the above development, we shall describe the process of image formation in an MSIIS.

Let there be $N$ spacecraft in the MSIIS. Let the positions of the spacecraft be denoted by $r_{1}(t), \cdots, r_{N}(t)$. Let

$$
\Delta r_{m n}(t)=r_{m}(t)-r_{n}(t), 1 \leq m, n \leq N .
$$

We assume that interferometric measurements are made between each pair of spacecraft. Consider an infinitesimal time interval, $[t, t+d t]$, and the incremental image accrued during this interval, $d I_{p}(Q, t)$. 
Then, using (13),

$$
d I_{p}(Q, t)=\frac{A^{2}}{\lambda z^{2}} \sum_{m=1}^{N} \sum_{n=1}^{N} \hat{J}\left(P_{m}, P_{n}\right) e^{\frac{2 \pi j}{\lambda z}\left(r\left(Q, P_{m}\right)-r\left(Q, P_{n}\right)\right)} d t
$$

where $\hat{J}\left(P_{m}, P_{n}\right)$ represents the noise corrupted measurement of the mutual intensity measurement between the spacecraft positions, $P_{m}$ and $P_{n}$, and $A$ denotes the area of the telescopes. Note that, $\hat{J}\left(P_{n}, P_{m}\right)=$ $\hat{J}^{*}\left(P_{m}, P_{n}\right)$ by definition. Let

$$
\hat{J}\left(P_{m}, P_{n}\right)=J\left(P_{m}, P_{n}\right)+\delta J_{m n}(t),
$$

where $\delta J_{m n}(t)$ is the noise corrupting the mutual intensity measurement between the $m^{t h}$ and the $n^{t h}$ spacecraft at instant $t$.

We also assume that the object we are imaging is spatially bounded, i.e., it has finite spatial dimensions. Thus, there exists a known $\Gamma \subseteq \Sigma_{I}$ such that the support of the image lies within $\Gamma$. Now, we introduce the "field-of-view" (FOV) or "picture frame" function, $A_{P}$, where

$$
A_{p}(Q)= \begin{cases}1, & Q \in \Gamma \\ 0, & Q \notin \Gamma\end{cases}
$$

In general, the support of $d I_{p}$ will not be within $\Gamma$. Thus, we crop the formed image $d I_{p}$ with the FOV function. Hence,

$$
\begin{aligned}
d I_{p}(Q, t)= & \frac{A^{2}}{\lambda z^{2}} \sum_{m=1}^{N} \sum_{n=1}^{N} A_{p}(Q) \hat{J}\left(P_{m}, P_{n}\right) e^{\frac{2 \pi j}{\lambda z}\left(r\left(Q, P_{m}\right)-r\left(Q, P_{n}\right)\right)} d t \\
& +\frac{A^{2}}{\lambda z^{2}} \sum_{m=1}^{N} \sum_{n=1}^{N} A_{p}(Q) \delta J_{m n}(t) e^{\frac{2 \pi j}{\lambda z}\left(r\left(Q, P_{m}\right)-r\left(Q, P_{n}\right)\right)} d t .
\end{aligned}
$$

At this point, we make a few simplifications using the fact that the distance between the image and the observation plane is very large compared to the other distances involved. Let $Q=\left(x_{Q}, y_{Q}, 0\right)$ and $P_{m}=$ $\left(x_{m}, y_{m}, z\right)$. Then,

$$
\begin{aligned}
& r\left(Q, P_{m}\right)=\sqrt{\left(x_{m}-x_{Q}\right)^{2}+\left(y_{m}-y_{Q}\right)^{2}+z^{2}} \\
& \simeq z\left[1+\frac{\left(x_{m}-x_{Q}\right)^{2}}{2 z^{2}}+\frac{\left(y_{m}-y_{Q}\right)^{2}}{2 z^{2}}\right] .
\end{aligned}
$$

Thus, it follows that

$$
r\left(Q, P_{m}\right)-r\left(Q, P_{n}\right) \approx \frac{1}{z}\left[\frac{1}{2}\left(x_{m}-x_{n}\right)\left(x_{m}-2 x_{Q}+x_{n}\right)+\frac{1}{2}\left(y_{m}-y_{n}\right)\left(y_{m}-2 y_{Q}+y_{n}\right)\right] .
$$

Also, it follows from the FHP that,

$$
J\left(P_{m}, P_{n}\right)=\frac{1}{\lambda^{2} z^{2}} \int_{\Sigma_{I}} I\left(Q^{\prime}\right) e^{\frac{2 \pi}{\lambda z}\left(r\left(Q^{\prime}, P_{m}\right)-r\left(Q^{\prime}, P_{n}\right)\right)} d S^{\prime} .
$$

Simplifying the exponents in (19) using (21) and substituting (22) in (19), we obtain the following equation,

$$
\begin{array}{r}
d I_{p}(Q, t)=\frac{A^{2}}{\lambda^{2} z^{2}} \sum_{m} \sum_{n} \frac{A_{p}(Q)}{\lambda^{2} z^{2}} \int_{\Sigma_{I}} I\left(Q^{\prime}\right) e^{\frac{2 \pi j}{\lambda z}\left[\Delta x_{m n}\left(x_{Q^{\prime}}-x_{Q}\right)+\Delta y_{m n}\left(y_{Q^{\prime}}-y_{Q}\right)\right]} d S^{\prime} d t+ \\
\frac{A^{2}}{\lambda^{2} z^{2}} \sum_{m} \sum_{n} A_{p}(Q) \delta J_{m n}(t) e^{\frac{-2 \pi j}{\lambda z}\left[\Delta x_{m n} x_{Q}+\Delta y_{m n} y_{Q}\right]} e^{-j \psi_{m n}(t)} d t,
\end{array}
$$

where

$$
\begin{gathered}
\psi_{m n}(t)=\frac{1}{\lambda z}\left(\rho_{m}^{2}-\rho_{n}^{2}\right), \\
\rho_{m}^{2}(t)=x_{m}^{2}(t)+y_{m}^{2}(t) .
\end{gathered}
$$

6 of 22 
Then, it follows that

$$
\begin{array}{r}
d I_{p}(Q, t)=\frac{A^{2}}{\lambda^{4} z^{4}} \sum_{m} \sum_{n} A_{p}(Q) \int_{\Sigma_{I}} I\left(Q^{\prime}\right) e^{\frac{2 \pi j}{\lambda z}\left[\Delta r_{m n} \cdot\left(Q^{\prime}-Q\right)\right]} d S^{\prime} d t+\cdots \\
\frac{A^{2}}{\lambda^{2} z^{2}} \sum_{m} \sum_{n} A_{p}(Q) \delta J_{m n}(t) e^{\frac{-2 \pi j}{\lambda z}[Q \cdot \Delta r]} e^{-j \psi_{m n}(t)} d t,
\end{array}
$$

where $\cdot$ denotes the standard scalar product in $\Re^{2}$. Recall the following Fourier transform identities: ${ }^{4}$

$$
\begin{array}{r}
\mathcal{F}(f * g)=\hat{f} \hat{g}, \\
\mathcal{F}\left(f(x) e^{-2 \pi j(a . x)}\right)=\hat{f}(\nu-a),
\end{array}
$$

where $\mathcal{F}$ and $*$ denotes the Fourier transform and the convolution operator respectively. Let

$$
H_{m n}(Q)=\int_{\Sigma_{I}} I\left(Q^{\prime}\right) e^{\frac{2 \pi j}{\lambda z}\left(\Delta r_{m n} \cdot\left(Q^{\prime}-Q\right)\right)} d S^{\prime} .
$$

Then, using the identities (27) and (28), and the relationship (29), taking the Fourier transform of both sides of (26), we obtain the following,

$$
\begin{array}{r}
d \hat{I}_{p}(\nu, t)=\left[\frac{A^{2}}{\lambda^{4} z^{4}} \sum_{m} \sum_{n} \hat{A}_{p}(\nu) * \hat{H}_{m n}(\nu)+\cdots\right. \\
\left.\frac{A^{2}}{\lambda^{2} z^{2}} \sum_{m} \sum_{n} \hat{A_{P}}\left(\nu-\frac{\Delta r_{m n}}{\lambda z}\right) \delta J_{m n}(t) e^{-j \psi_{m n}(t)}\right] d t .
\end{array}
$$

In the above equation, all the hatted variables represent the Fourier transforms of their unhatted counterparts. Again, due to the Fourier identities, it follows that

$$
\hat{H}_{m n}(\nu)=\hat{I}(\nu) \delta\left(\nu-\frac{\Delta r_{m n}}{\lambda z}\right) .
$$

Substituting (31) in (30), we use the sampling property of the delta function to obtain

$$
\begin{array}{r}
d \hat{I}_{p}(\nu, t)=\left[\frac{A^{2}}{\lambda^{4} z^{4}} \sum_{m} \sum_{n} \hat{A}_{p}\left(\nu-\frac{\Delta r_{m n}}{\lambda z}\right) \hat{I}\left(\frac{\Delta r_{m n}}{\lambda z}\right)+\cdots\right. \\
\left.\frac{A^{2}}{\lambda^{2} z^{2}} \sum_{m} \sum_{n} \hat{A_{P}}\left(\nu-\frac{\Delta r_{m n}}{\lambda z}\right) \delta J_{m n}(t) e^{-j \psi_{m n}(t)}\right] d t .
\end{array}
$$

Hence, the frequency content of the final picture is given by the following equation,

$$
\begin{array}{r}
\hat{I_{p}}(\nu, t)=\frac{A^{2}}{\lambda^{4} z^{4}} \int_{0}^{T} \sum_{m} \sum_{n} \hat{A}_{p}\left(\nu-\frac{\Delta r_{m n}}{\lambda z}\right) \hat{I}\left(\frac{\Delta r_{m n}}{\lambda z}\right) d t+\cdots \\
\frac{A^{2}}{\lambda^{2} z^{2}} \sum_{m} \sum_{n} \int_{0}^{T} \hat{A_{P}}\left(\nu-\frac{\Delta r_{m n}}{\lambda z}\right) \delta J_{m n}(t) e^{-j \psi_{m} n(t)} d t,
\end{array}
$$

where $T$ denotes the total time of the imaging maneuver for the MSIIS.

Since $z$ is large, $I\left(P_{1}\right) \approx I\left(P_{2}\right) \forall P_{1}, P_{2} \in \Sigma_{o}$ (as follows directly from the HFP). Let

$$
I_{e s t}=I(P), P \in \Sigma_{o},
$$

i.e., $I_{e s t}$ is the intensity of radiation at the observation plane. We have

$$
I_{e s t}=\frac{\hat{I}(0)}{\lambda^{2} z^{2}} .
$$


Note that a good estimate of $I_{\text {est }}$ would be available at the end of the imaging maneuver. Then, multiplying (33) by the factor $\frac{\lambda^{2} z^{2}}{A^{2} \hat{A}_{p}(0) I_{e s t}}$, we have the normalized equation

$$
\begin{array}{r}
\hat{\mathbf{I}}_{p}(\nu)=\int_{0}^{T} \sum_{m} \sum_{n} \hat{\mathbf{A}}_{p}\left(\nu-\frac{\Delta r_{m n}}{\lambda z}\right) \hat{\mathbf{I}}\left(\frac{\Delta r_{m n}}{\lambda z}\right) d t+\cdots \\
\frac{1}{I_{e s t}} \int_{0}^{T} \sum_{m} \sum_{n} \hat{\mathbf{A}}_{p}\left(\nu-\frac{\Delta r_{m n}}{\lambda z}\right) \delta J_{m n} e^{-j \psi_{m} n(t)} d t .
\end{array}
$$

In the above equation,

$$
\begin{array}{r}
\hat{\mathbf{A}}_{p}(\nu)=\frac{\hat{A}_{p}(\nu)}{\hat{A}_{p}(0)}, \\
\hat{\mathbf{I}}(\nu)=\frac{\hat{I}(\nu)}{\hat{I}(0)}, \\
\hat{\mathbf{I}}_{p}(\nu)=\frac{\lambda^{2} z^{2}}{A^{2} \hat{A}_{p}(0) I_{e s t}} \hat{I}(\nu) .
\end{array}
$$

At this point, we make the following approximation,

$$
\int_{0}^{T} \hat{\mathbf{A}}_{p}\left(\nu-\frac{\Delta r_{m n}}{\lambda z}\right) \hat{\mathbf{I}}\left(\frac{\Delta r_{m n}}{\lambda z}\right) d t \approx \int_{0}^{T} \hat{\mathbf{A}}_{p}\left(\nu-\frac{\Delta r_{m n}}{\lambda z}\right) \hat{\mathbf{I}}(\nu) d t .
$$

The justification of the above approximation is as follows. The shape of $\hat{A}_{p}$ is shown in Fig. 4.3 for a circular FOV function. Note that the values of $\hat{\mathbf{A}}_{p}(\nu)$ are high (of the order of 1 ) close to $\nu=0$, and drop off sharply for values of $\nu$ further out. Thus, it is reasonable to expect that the major contribution to the left hand side in $(40)$ is due to the time instants when $\left(\nu-\frac{\Delta r_{m n}}{\lambda z}\right) \approx 0$. We know that the object being imaged is spatially bounded and that the spectra of spatially bounded images are analytic functions in the spatial frequency plane. ${ }^{11}$ Thus, it follows that the spectrum of the image is continuous. Thus, if $\left(\nu-\frac{\Delta r_{m n}}{\lambda z}\right) \approx 0$, then $\hat{\mathbf{I}}\left(\frac{\Delta r_{m n}}{\lambda z}\right) \approx \hat{\mathbf{I}}(\nu)$. Hence, we can make the approximation in (40). Then, assuming that the approximation holds, we have:

$$
\hat{\mathbf{I}}_{p}(\nu)=M(\nu) \hat{\mathbf{I}}(\nu)+N(\nu)
$$

where

$$
\begin{array}{r}
M(\nu)=\int_{0}^{T} \sum_{m} \sum_{n} \hat{\mathbf{A}}_{p}\left(\nu-\frac{\Delta r_{m n}}{\lambda z}\right) d t \\
N(\nu)=\frac{1}{I_{e s t}} \int_{0}^{T} \sum_{m} \sum_{n} \hat{\mathbf{A}}_{p}\left(\nu-\frac{\Delta r_{m n}}{\lambda z}\right) \delta J_{m n} e^{-j \psi_{m n}(t)} d t .
\end{array}
$$

Equation (41) describes the equivalent optical instrument that is formed by the imaging maneuver of an MSIIS; $M(\nu)$ is the modulation transfer function of the synthesized optical aperture and $N(\nu)$ represents the noise corrupting the measurement (in the frequency domain). Note that both the MTF and the noise corrupting the optical instrument are dependent on the trajectory of the spacecraft. In the next section, we shall formulate a detailed model of the noise corrupting the image in an MSIIS.

\section{Modeling of the Noise Process in an MSIIS}

In this section, we shall formulate a model for the noise corrupting the image formed by an MSIIS. Specifically, we shall show that the noise is dependent on the arrival rate of the photons from the source at the observation plane. From the previous section, we know that the noise corrupting the image formed by the MSIIS at spatial frequency $\nu$ is given by the relationship,

$$
N(\nu)=\frac{1}{I_{e s t}} \int_{0}^{T} \sum_{m} \sum_{n} \hat{\mathbf{A}}_{p}\left(\nu-\frac{\Delta r_{m n}}{\lambda z}\right) \delta J_{m n} e^{-j \psi_{m n}(t)} d t .
$$


As noted before, $\delta J_{m n}$ is the noise in the mutual intensity measurement made between the $m^{t h}$ and the $n^{t h}$ spacecraft at time $t$. Consider the infinitesimal time interval $[t, t+d t]$. Here we note that $\delta J_{m n}$, in general, is a complex random variable. Let us denote its real and imaginary parts by $\delta J_{m n}^{r}(t)$ and $\delta J_{m n}^{i}(t)$ respectively.

The light collected from the two spacecraft is interfered to form a fringe, which is detected using a photodetector array. For a detailed description of such a system, please refer to. ${ }^{11}$ Let the number of detectors in the array be denoted by $M$ and let the area of each element be denoted by $d A$. It is known that (see chapter 9 , section 4 , pg. 490-501 in ${ }^{11}$ ):

$$
\begin{array}{r}
E\left[\delta J_{m n}^{r}\right]=0, \\
\operatorname{var}\left[\delta J_{m n}^{r}\right]=\frac{e}{M d A d T} I_{e s t},
\end{array}
$$

where $e=\frac{h c}{\lambda}$ denotes the energy of the photons incident on the photodetectors, and $\operatorname{var}[x]$ denotes the variance of the random variable $x$. Similarly,

$$
\begin{array}{r}
E\left[\delta J_{m n}^{i}\right]=0 \\
\operatorname{var}\left[\delta J_{m n}^{i}\right]=\frac{e}{M d A d T} I_{\text {est }} .
\end{array}
$$

Furthermore, it is known that,

$$
E\left[\delta J_{m n}^{r}(t) \delta J_{m n}^{i}(t)\right]=0 .
$$

If the total number of photon arrivals across the photodetector array is large enough, then due to the central limit theorem, it follows that $\delta J_{m n}^{r}$ and $\delta J_{m n}^{i}$ are both Gaussian random variables. Furthermore, since they are uncorrelated, it follows that they are independent. Let

$$
A_{\text {tot }}=M d A \text {. }
$$

Thus, it follows that

$$
\begin{aligned}
\delta J_{m n}^{r}(t) & \sim N\left(0, \frac{e I_{\text {est }}}{A_{t o t} d T}\right), \\
\delta J_{m n}^{i}(t) & \sim N\left(0, \frac{e I_{e s t}}{A_{t o t} d T}\right),
\end{aligned}
$$

where $N(\mu, \sigma)$ denotes a Gaussian random variable with mean $\mu$, and variance $\sigma$. We define

$$
N_{m n}(\nu)=\frac{1}{I_{e s t}} \int_{0}^{T} \hat{\mathbf{A}}_{p}\left(\nu-\frac{\Delta r_{m n}}{\lambda z}\right) \delta J_{m n} e^{-j \psi_{m n}(t)},
$$

i.e., the noise at spatial frequency $\nu$ due to the $m^{\text {th }}$ and $n^{\text {th }}$ spacecraft pair. Let

$$
T=L d t
$$

Then, we can approximate $N_{m n}(\nu)$ as follows

$$
N_{m n}(\nu) \approx \frac{1}{I_{e s t}} \sum_{k=1}^{L} \hat{\mathbf{A}}_{p}\left(\nu-\frac{\Delta r_{m n}^{k}}{\lambda z}\right) \delta J_{m n}^{k} e^{-j \psi_{m n}^{k}} d t,
$$

where

$$
\begin{gathered}
\delta J_{m n}^{k}=\delta J_{m n}(k d t), \\
\psi_{m n}^{k}=\psi_{m n}(k d t), \\
\Delta r_{m n}^{k}=\Delta r_{m n}(k d t) .
\end{gathered}
$$

Noting that $\delta J_{m n}^{k}$ and $\delta J_{m n}^{l}$ are independent random variables whenever $k \neq l$, and that $e^{\psi_{m n}^{k}}$ is a pure phase term, it follows that $N_{m n}(\nu)$ is a complex zero-mean Gaussian random variable with variance given by

$$
\operatorname{var}\left[N_{m n}(\nu)\right]=\frac{2 e}{I_{e s t} A_{t o t}} \sum_{k=1}^{L}\left|\hat{\mathbf{A}}_{p}\left(\nu-\frac{\Delta r_{m n}}{\lambda z}\right)\right|^{2} d t
$$


Again, note that $N_{m n}(\nu)$ is independent of $N_{p q}(\nu)$, unless $m=q$ and $n=p$, or $m=p$ and $n=q$. However, we have that

$$
\hat{\mathbf{A}}_{p}\left(\nu-\frac{\Delta r_{m n}}{\lambda z}\right) \hat{\mathbf{A}}_{p}\left(\nu+\frac{\Delta r_{m n}}{\lambda z}\right) \approx 0
$$

and hence, we have the following equation:

$$
\operatorname{var}[N(\nu)]=\frac{2 e}{I_{e} A_{t o t}} \sum_{m} \sum_{n}\left(\sum_{k=1}^{L}\left|\hat{\mathbf{A}}_{p}\left(\nu-\frac{\Delta r_{m n}}{\lambda z}\right)\right|^{2} d t\right) .
$$

Noting that $I_{\text {est }}=e \mu$, where $\mu$ is the average arrival rate of photons on the measurement plane, it follows that

$$
\operatorname{var}[N(\nu)]=\frac{2}{\mu_{t o t}} \sum_{m} \sum_{n}\left(\sum_{k=1}^{L}\left|\hat{\mathbf{A}}_{p}\left(\nu-\frac{\Delta r_{m n}}{\lambda z}\right)\right|^{2} d t\right),
$$

where $\mu_{t o t}=\mu A_{t o t}$, i.e., the average arrival rate of the photons over the entire detection area. Thus, as $d t \rightarrow 0$, we can write the noise corrupting the image at spatial frequency $\nu$ as follows:

$$
N(\nu)=\sqrt{\frac{2}{\mu_{t o t}}} \int_{0}^{T} \sum_{m} \sum_{n} \hat{\mathbf{A}}_{p}\left(\nu-\frac{\Delta r_{m n}}{\lambda z}\right) \delta \bar{J}_{m n}(t) d t,
$$

where $\delta J_{m n}(t)$ is a unit variance circular Gaussian White noise process. Thus, we have characterized the nature of the noise corrupting the image formed in an MSIIS. In the next section, we shall list a few of the salient features of the image model which was formulated for the process of image formation in an MSIIS.

\section{Properties of the Imaging Model of an MSIIS}

The final image formed by an MSIIS can be written as follows (in the Spatial frequency domain):

$$
\hat{\mathbf{I}}_{p}(\nu)=M(\nu) \hat{\mathbf{I}}(\nu)+N(\nu)
$$

where

$$
\begin{array}{r}
M(\nu)=\int_{0}^{T} \sum_{m} \sum_{n} \hat{\mathbf{A}}_{p}\left(\nu-\frac{\Delta r_{m n}}{\lambda z}\right) d t \\
N(\nu)=\sqrt{\frac{2}{\mu_{t o t}}} \int_{0}^{T} \sum_{m} \sum_{n} \hat{\mathbf{A}}_{p}\left(\nu-\frac{\Delta r_{m n}}{\lambda z}\right) \delta \bar{J}_{m n}(t) d t .
\end{array}
$$

We make the following observations about the imaging model that is formulated above:

- The MTF, $M(\nu)$, and the noise corrupting the image formed by the MSIIS, $N(\nu)$, are functions of the spacecraft trajectory.

- The noise magnitude is inversely proportional to the square root of the arrival rate of the photons from the source on the measurement plane. Also, the noise magnitude is inversely proportional to the square root of the collection area of the telescopes. Thus, the noise can be reduced by increasing the collection area of the telescopes. However, note that costs (and other possible issues such as fuel usage) often dictate the size of the telescopes that can be put in space.

- Consider the image model of an MSIIS, (64). We can obtain an estimate of the image by dividing throughout by $M(\nu)$. Then, the noise corrupting the estimated image is given by

$$
e(\nu)=\frac{N(\nu)}{M(\nu)} .
$$


It can be seen that $e(\nu)$ is zero-mean circular Gaussian with variance given by

$$
\begin{array}{r}
\operatorname{var}[e(\nu)]=\frac{E|N(\nu)|^{2}}{|M(\nu)|^{2}} \\
=\frac{2}{\mu_{t o t}} \frac{\int_{0}^{T} \sum_{m} \sum_{n}\left|\hat{\mathbf{A}}_{p}\left(\nu-\frac{\Delta r_{m n}}{\lambda z}\right)\right|^{2} d t}{\left|\int_{0}^{T} \sum_{m} \sum_{n} \hat{\mathbf{A}}_{p}\left(\nu-\frac{\Delta r_{m n}}{\lambda z}\right) d t\right|^{2}} .
\end{array}
$$

Also, note that $\hat{\mathbf{A}}_{p}\left(\nu-\frac{\Delta r_{m n}}{\lambda z}\right) \approx 0$, except when $\left(\nu-\frac{\Delta r_{m n}}{\lambda z}\right) \approx 0$. Thus, the above expression can be approximated as

$$
\operatorname{var}[e(\nu)]=\frac{1}{\mu_{t o t}} \frac{1}{\int_{0}^{T} \sum_{m} \sum_{n} \hat{\mathbf{A}}_{p}\left(\nu-\frac{\Delta r_{m n}}{\lambda z}\right) d t} .
$$

Note that the above is a "Noise to Signal Ratio" in the sense that it is a measure of the noise corrupting the final reconstructed image. Further, the denominator of the above expression is equal to the number of photons that are collected at the spatial frequency, $\nu$. Thus, the "Signal to Noise" ratio at a spatial frequency is equal to the number of photons that are collected at that spatial frequency. Note that the frequency content of an image is evaluated by forming fringes at various spatial frequencies. The above result states that the accuracy, or the signal to noise ratio, of the fringe formed, is directly proportional to the number of photons used to form the fringe.

- Let us consider the instantaneous situation over the Fourier domain in the infinitesimal time interval, $[t, t+d t]$. The MSIIS is equivalent to an optical filter, whose MTF is given by

$$
d M(\nu)=\sum_{m} \sum_{n} \hat{\mathbf{A}}_{p}\left(\nu-\frac{\Delta r_{m n}}{\lambda z}\right) d t .
$$

If we approximate the function $\hat{\mathbf{A}}_{p}$ by a disk function, i.e., a function whose support is the circular region in which, $\hat{\mathbf{A}}_{p}(\nu) \approx 1$, then the instantaneous picture is shown in Fig. 4.4. If we assume that the frequency content of the image is mostly concentrated in the region $\Omega$, (which we would know from the resolution requirements), then the build-up of the MTF of the optical aperture can be likened to the painting of a "big disk" or "resolution disk" by a number of smaller "paintbrushes" or "coverage disks". Typically, the ratio of the radii of the big disk to the coverage disks is equal to the number of pixels desired in the image. Referring to (69), we observe that the variance of the noise at any spatial frequency is inversely proportional to the "thickness" of the paint laid down at that frequency. Hence, in order to take a good image, the scaled relative trajectories of the constituent spacecraft (scaled by the factor $\lambda \bar{z}$ ), need to be dense to a radius $\rho$, (the radius of the coverage disks), within the resolution disk $\Omega$. Mathematically, this means that for at least one pair of spacecrafts, $(m, n)$,

$$
\left\|\frac{\Delta r_{m n}}{\lambda z}-\nu\right\| \leq \rho, \forall \nu \in \Omega
$$

where $\|$.$\| denotes the \Re^{2}$ Euclidean norm, $\rho=R / N$, (in the case of an $\mathrm{N} \times \mathrm{N}$ image), and $R$ is the radius of the region $\Omega$.

Also, the paint laid down at any spatial frequency $\nu$ is equal to the MTF accrued by the spacecraft trajectories at that frequency and is equal to the time spent by the scaled relative trajectories within radius $\rho$ of the spatial frequency point. Hence, the relative trajectories need to be "slow" enough so that the requisite signal to noise ratio can be achieved at every spatial frequency point, i.e.,

$$
M(\nu) \geq \Delta, \forall \nu \in \Omega
$$

The issue of the minimum level of signal noise ratio, $\Delta$, required is addressed in. ${ }^{6}$ In the next section, we give a numerical example where we illustrate the pitfalls in imaging without ensuring that the relative trajectories of the constituent spacecraft are dense enough in the region $\Omega$. 


\section{Imaging Example}

In this section, we present an imaging example where we obtain a $100 \times 100$ image of a Jupiter-sized planet at a distance of 8 Parsecs. The radius of the region $\Omega$ can be found using the Rayleigh two-point resolution criterion. ${ }^{4}$ However, we can scale the problem such that $R=1$, where $R$ is the radius of the resolution disk. Note that, as mentioned before, $\rho=R / N$, where the image is $\mathrm{N}$ x $\mathrm{N}$ pixels. Thus in this case, $N=100$. In this example, we assume that we are imaging using a four spacecraft system. It has been argued in ${ }^{7,9,10}$ that spiral trajectories might be the most efficient way of covering the spatial frequency plane. In fact, it has been shown in ${ }^{7,8}$ that they are time optimal. However, care needs to be taken to ensure that these spiral trajectories are dense enough so that they cover the resolution disk without leaving gaps. In the following example, we compare the imaging performance of two spiral trajectories: trajectory 1 is tight enough such that the spatial frequency is covered densely to radius $\rho$ by the relative trajectories of the constituent spacecraft and Trajectory 2 is a looser spiral which does not satisfy the denseness condition of the trajectories, as required by (71). In Figs. $5-8$, we show the time history of image formation for Trajectory 1 . The top left hand corner of the plots shows the spatial frequency plane coverage, the top right hand corner plot shows the formed image and the bottom left hand corner plot shows the actual image. In Figs. 9 - 12, we show the same for Trajectory 2. It can be seen that the performance of Trajectory 1 is far superior to that of Trajectory 2. Hence, this illustrates that the spacecraft relative trajectory need to be dense enough for good imaging performance. Also, they need to be slow enough such that enough signal to noise ratio is built up so that the reconstructed image is good enough, as required by (72).

\section{Conclusions}

In this paper, we have obtained a detailed model for the process of image formation in an MSIIS. We have shown that the MSIIS can be described by an equivalent optical instrument. We have shown that the MTF of the synthesized instrument, and the noise corrupting the final image in an MSIIS are functions of the trajectories of the constituent spacecraft. Moreover, we have introduced the notion of image formation by an MSIIS being analogous to the "painting" of a big disk by a number of smaller "coverage disks" or "paintbrushes". This is also the first attempt at the modeling of the "dynamics" of image formation in MSIIS. We have shown that satisfactory imaging corresponds to the spacecraft relative trajectories being "dense" enough in a given region while ensuring that they are "slow" enough. These requirements have been explicitly quantified. Furthermore, it would be reasonable to expect that the critical paint thickness for satisfactory imaging performance would be dependent on the application at hand, i.e., the questions that the image would be used to answer. ${ }^{6}$ The solution to the time optimization problem posed in this paper is addressed in..$^{7,8}$ However, the problem of fuel minimization while satisfying the imaging constraints is an open question and is a subject of our current research.

\section{References}

${ }^{1}$ http://origins.jpl.nasa.gov/missions/pi.html

${ }^{2}$ A. Quirrenbach, "Optical Inteferometry", Annual Review in Astronomy and Astrophysics, 39, 2001, pp 353-401.

${ }^{3}$ W. J. Borucki, D. G. Koch, E. W. Dunham, J. M. Jenkins," The Kepler Mission : A Mission to Determine the Frequency of Inner Planets Near the Habitable zone of a Wide Range of Stars", presented at Planets beyond our Solar System and Next Generation Space Missions, ASP conference series, 16-18 Oct. 1996

${ }^{4}$ J. W. Goodman, Introduction to Fourier Optics, Boston, MA, McGraw Hill, 1996

${ }^{5}$ J. P. Fitch,Synthetic Aperture Radar, Springer-Verlag,Berlin,1987

${ }^{6}$ S. Chakravorty, P. T. Kabamba and D. C. Hyland, "Guaranteed Classification Performance of Multi-Spacecraft Interferometric Imaging Systems", Journal of the Astronautical Sciences, Vol.51, No. 2, April-June 2003, pp. 205-226

${ }^{7}$ S. Chakravorty, Design and Optimal Control of Multi-Spacecraft Interferometric Imaging Systems, PhD thesis, Department of Aerospace Engineering, University of Michigan, 2004

${ }^{8}$ S. Chakravorty, P. T. Kabamba and D. C. Hyland, "Design of Minimum Time Maneuvers for Multi-Spacecraft Interferometric Imaging Systems", in review for The Journal of the Astronautical Sciences

${ }^{9}$ E M C Kong, D W Miller and R J Sedwick," Exploiting Orbital dynamics for Aperture Synthesis using Distributed Satellite Systems: Applications to Visible Earth Imager System", Journal Astronautical Sciences, v 47 n 1-2, 1999, 53-75

${ }^{10}$ A. B. DeCue, " Orbital station-keeping for multiple spacecraft interferometry", Journal of the Astronautical Sciences, vol. 39, no. 3, 1991, 283-297.

${ }^{11}$ J. W. Goodman, "Statistical Optics", Wiley Classics Library, Wiley, 1996 


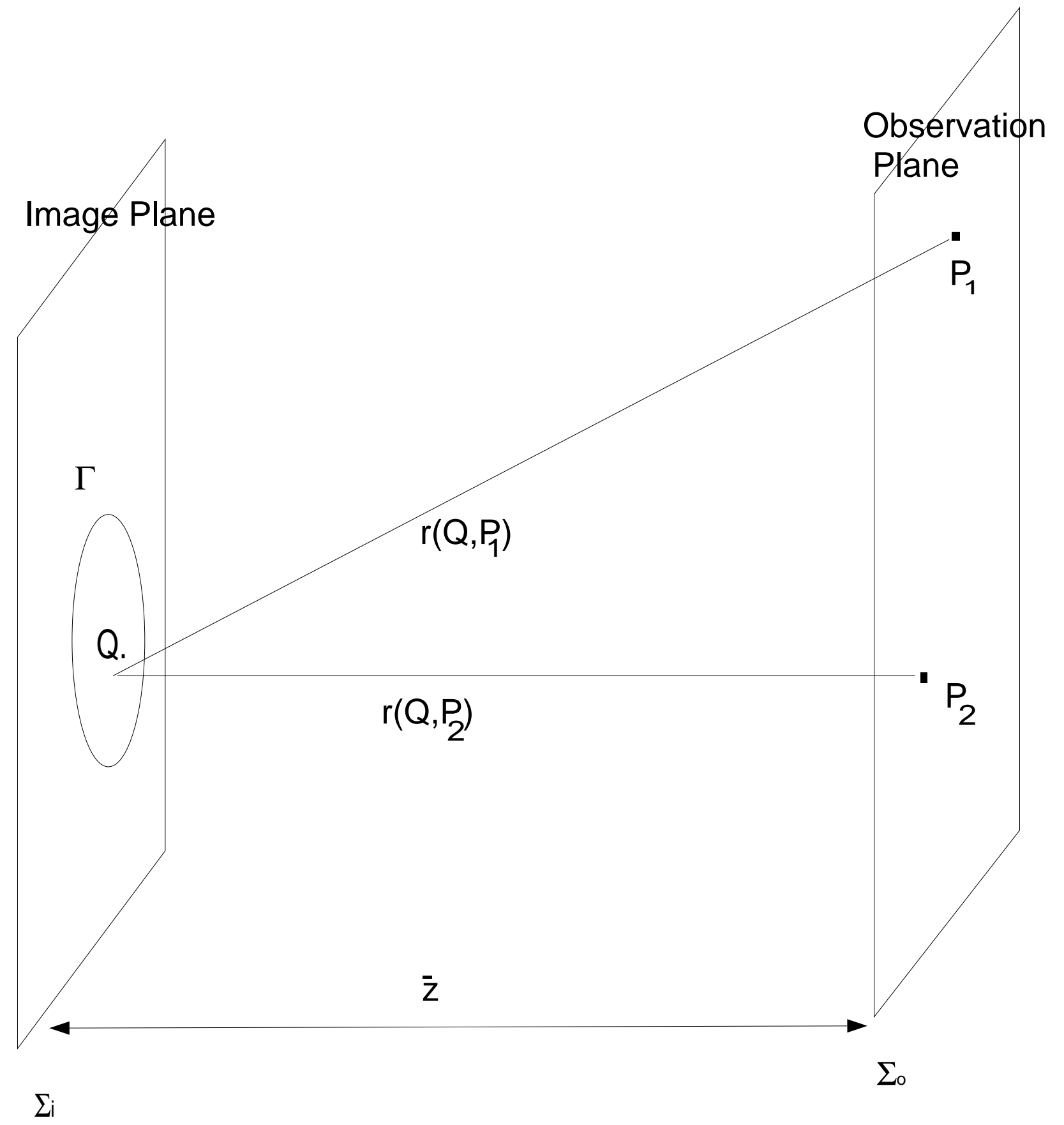

Figure 2. Propagation of Mutual Intensity from the Image Plane to Measurement Plane

13 of 22 


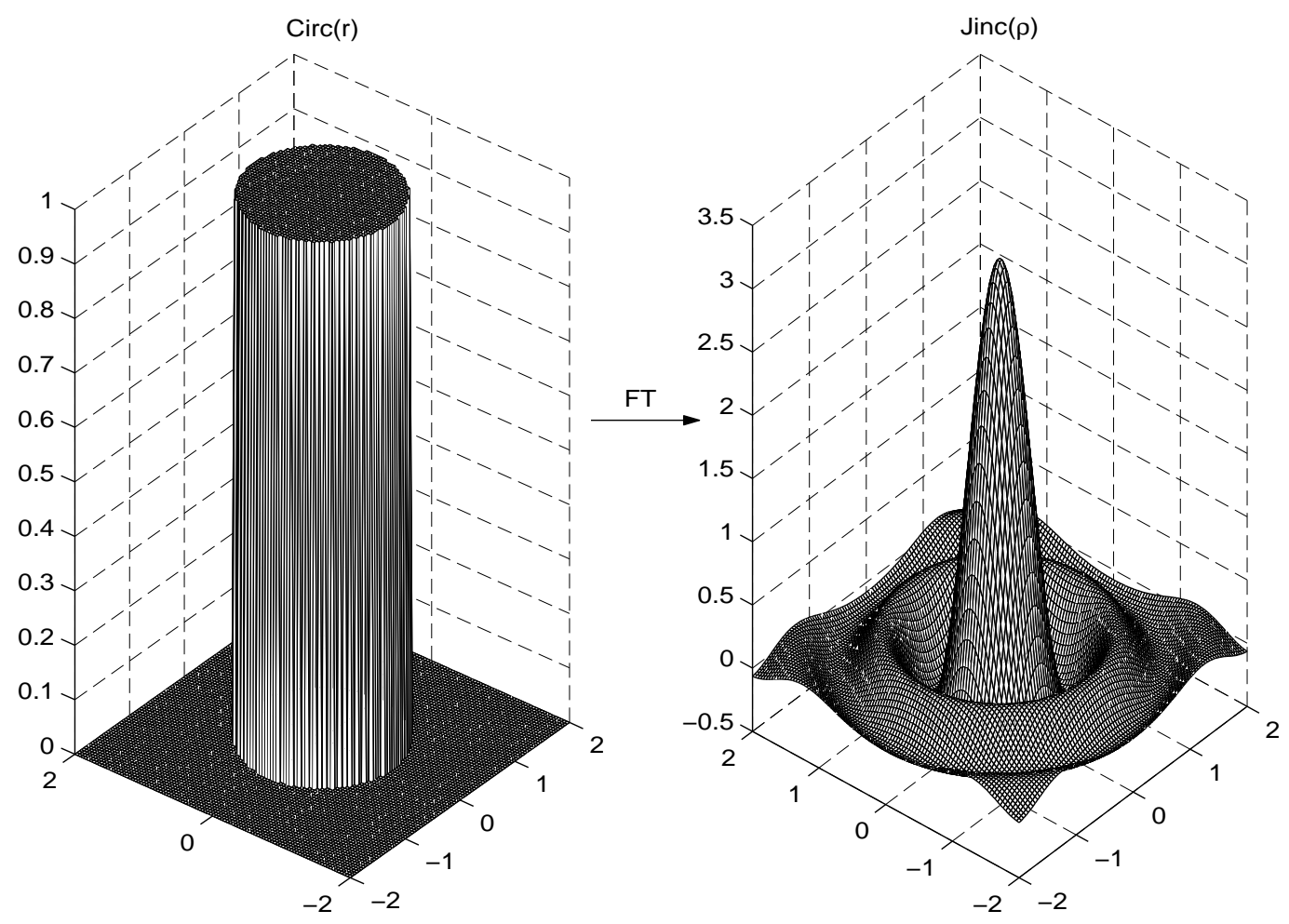

Figure 3. FOV Function and its Fourier Transform
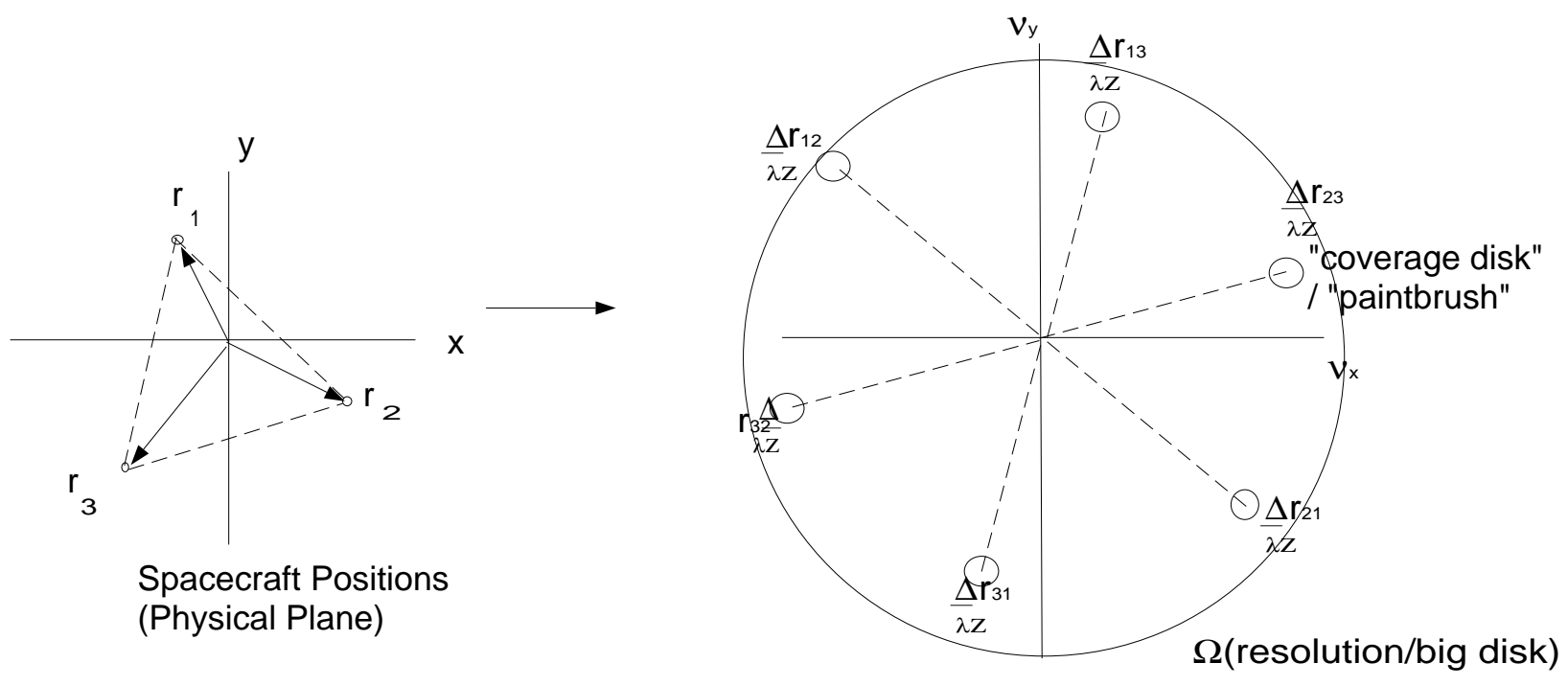

Spatial Frequency Coverage

Figure 4. Imaging as Painting

\section{4 of 22}



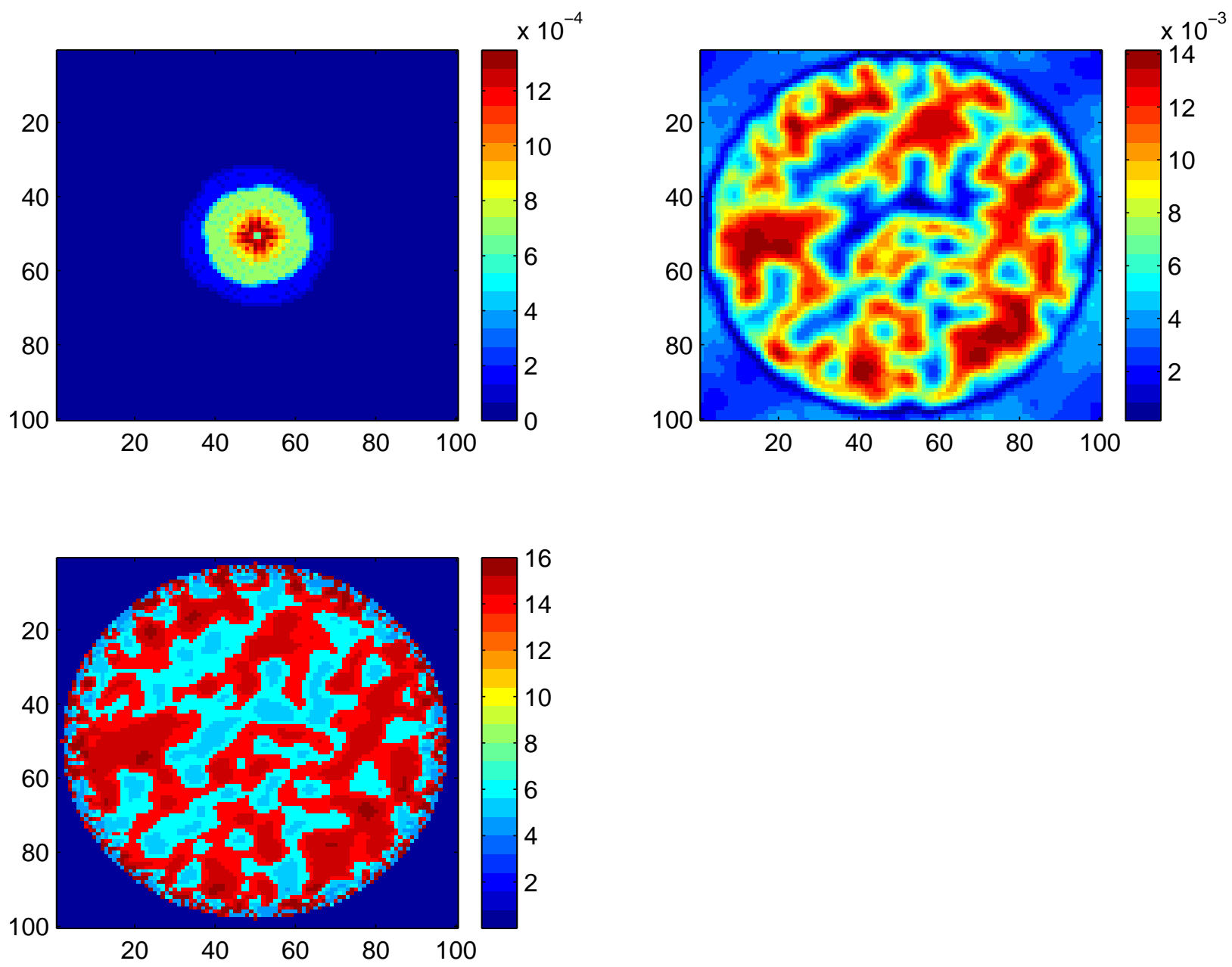

Figure 5. Image formed at $0.05 T_{f}$ by Trajectory 1 

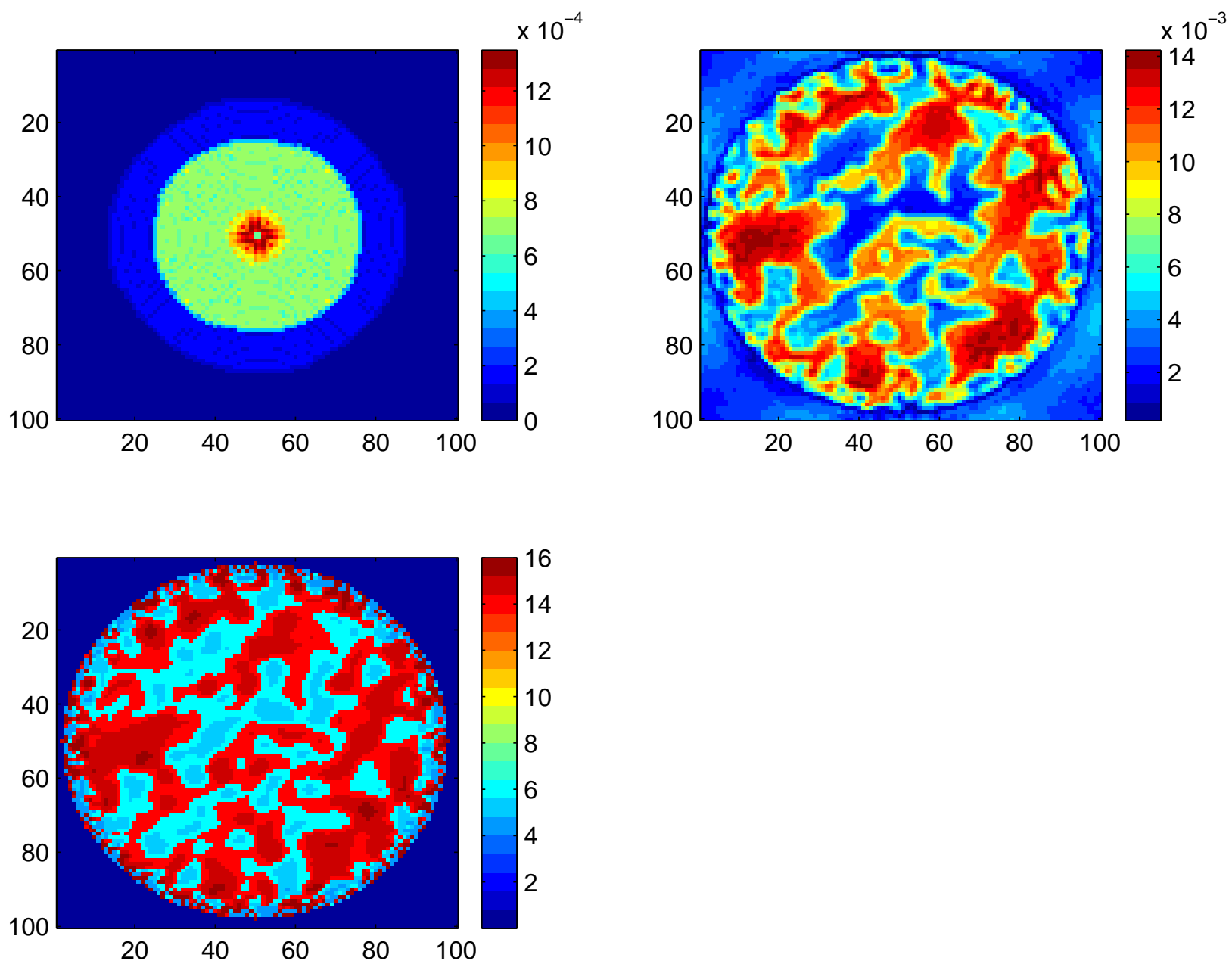

Figure 6. Image formed at $0.20 T_{f}$ by Trajectory 1 

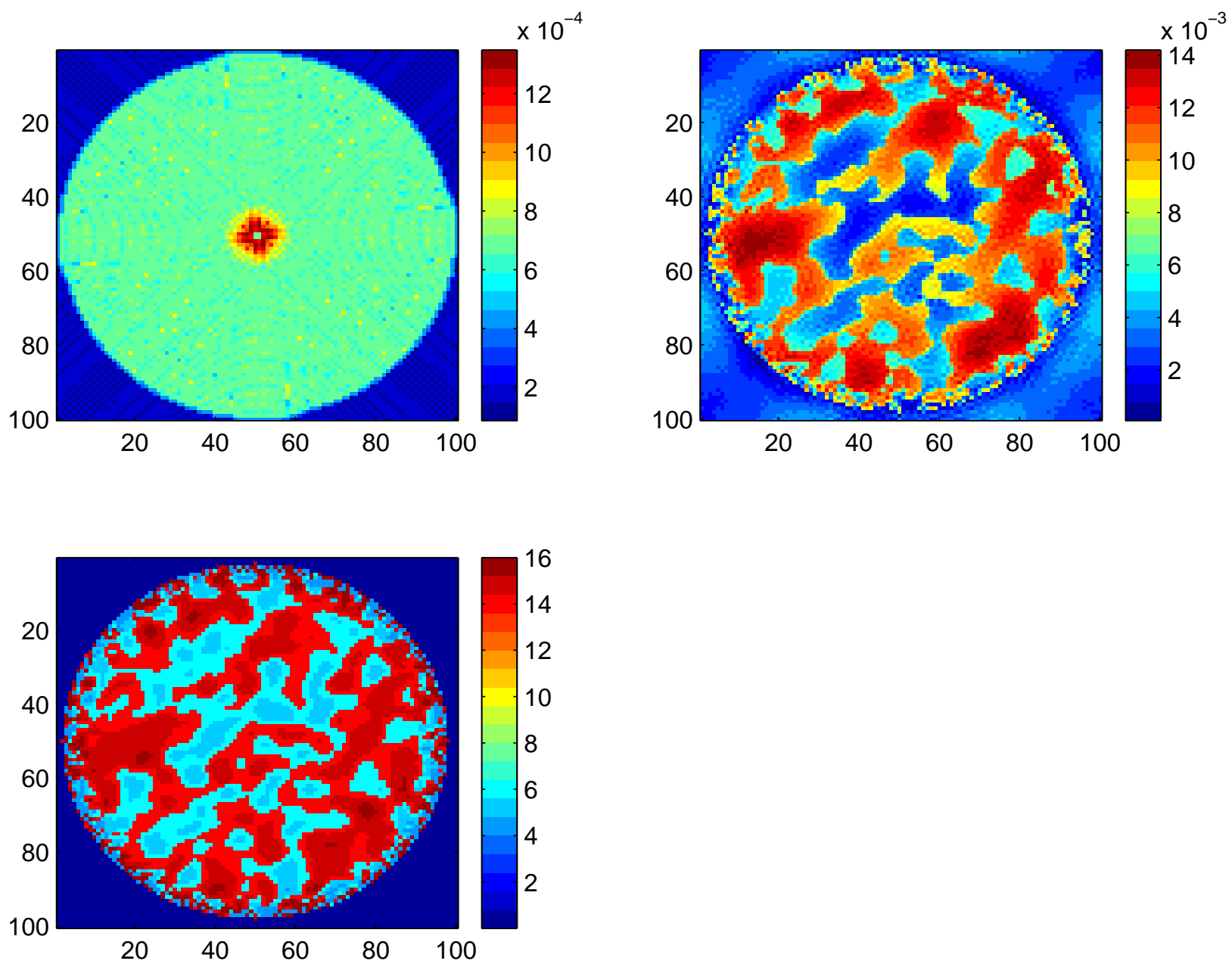

Figure 7. Image formed at $0.5 T_{f}$ by Trajectory 1 

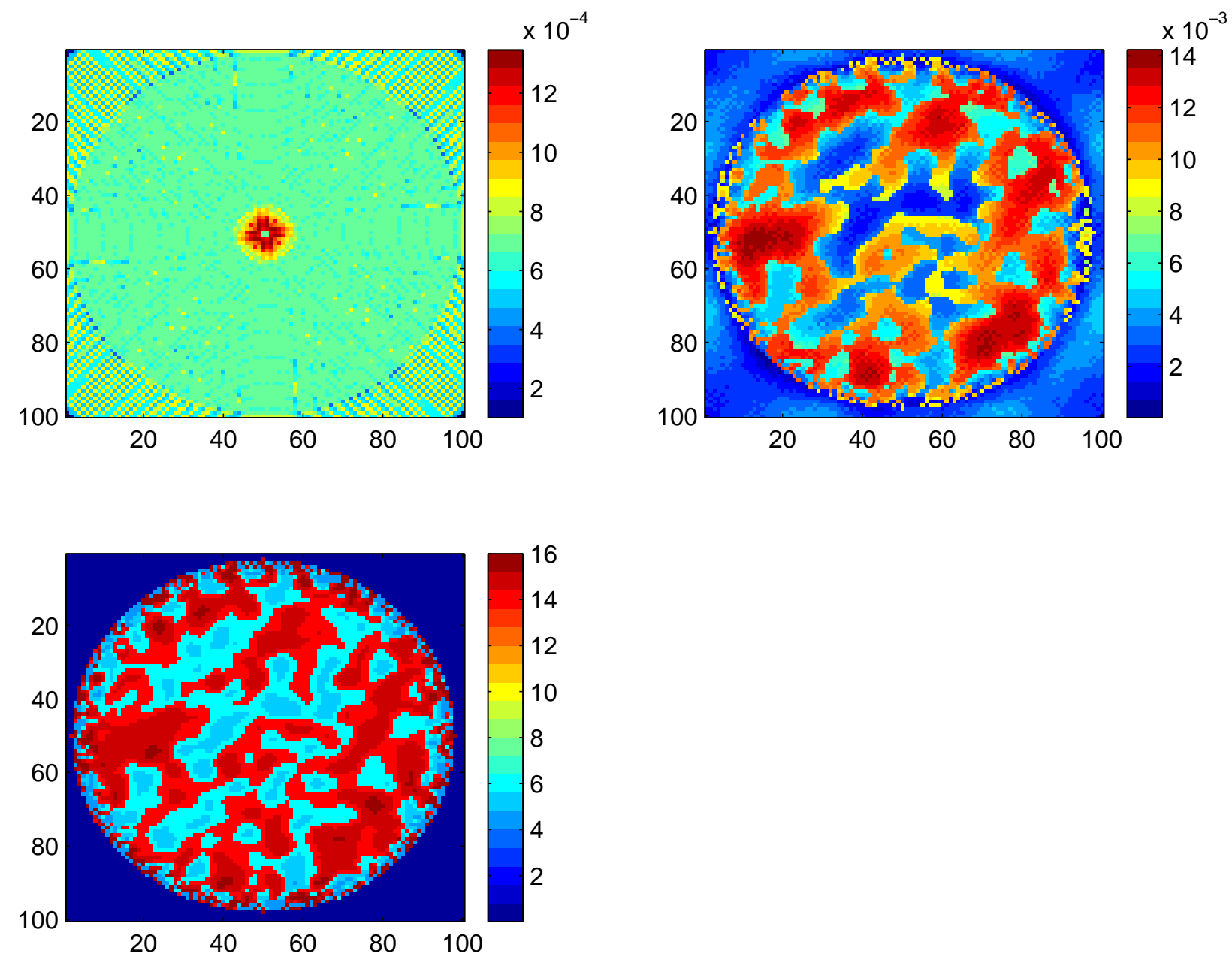

Figure 8. Imaging formed at $T_{f}$ by Trajectory 1 


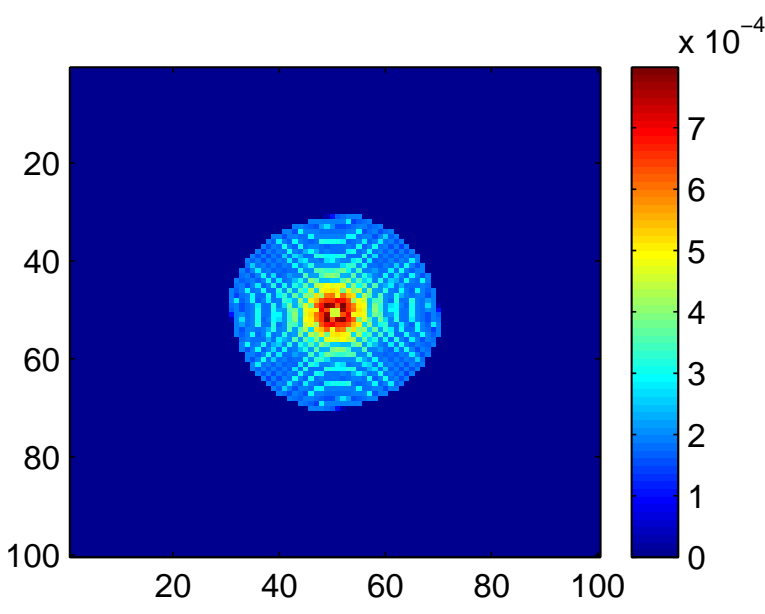

spatial frequency coverage

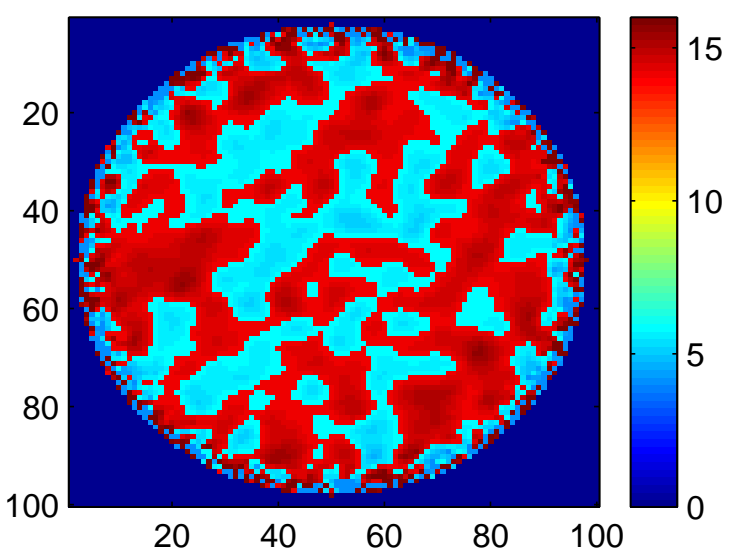

Actual Image

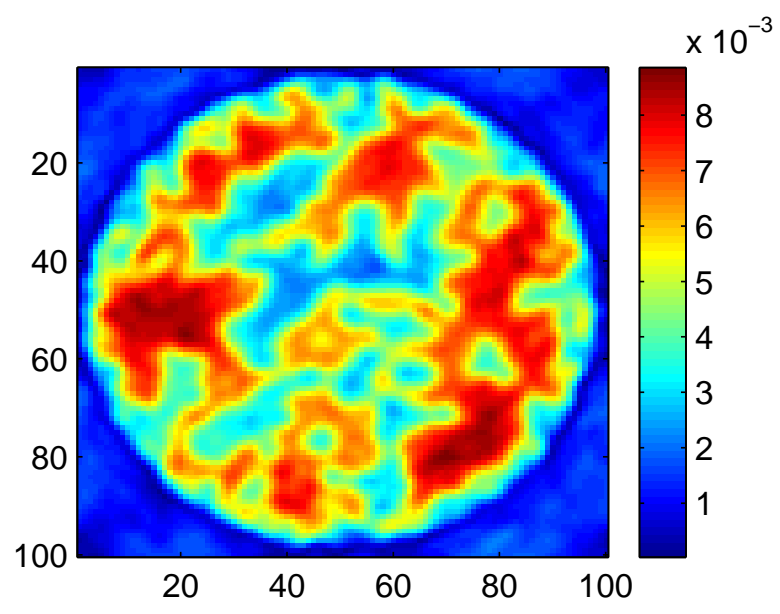

Formed Image

Figure 9. Image formed at $0.05 T_{f}$ by Trajectory 2 


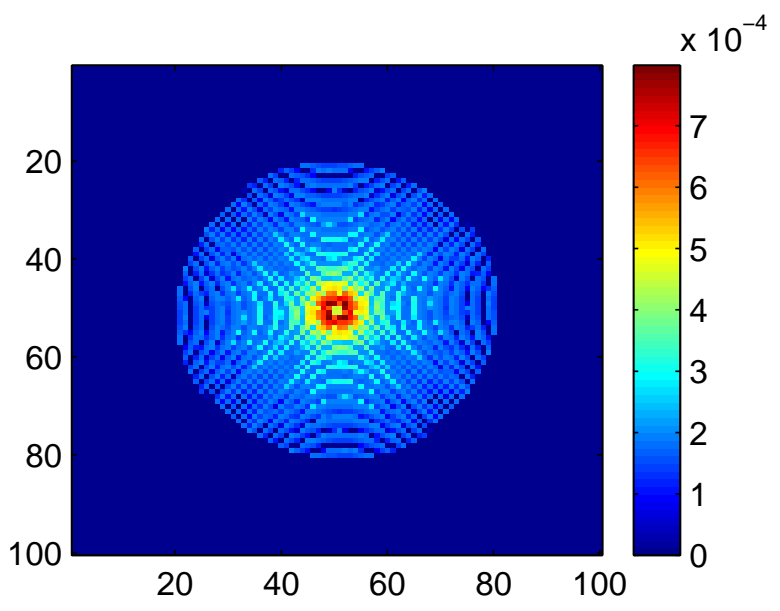

Spatial Frequency Coverage

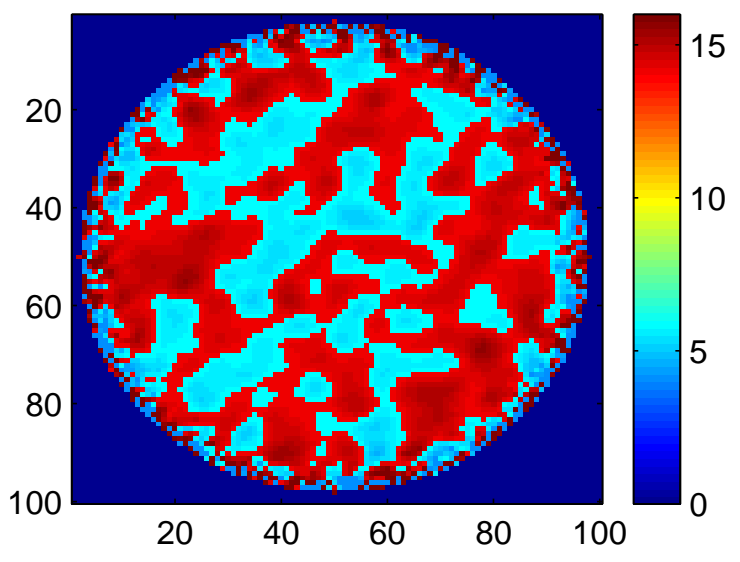

Actual Image

5

10

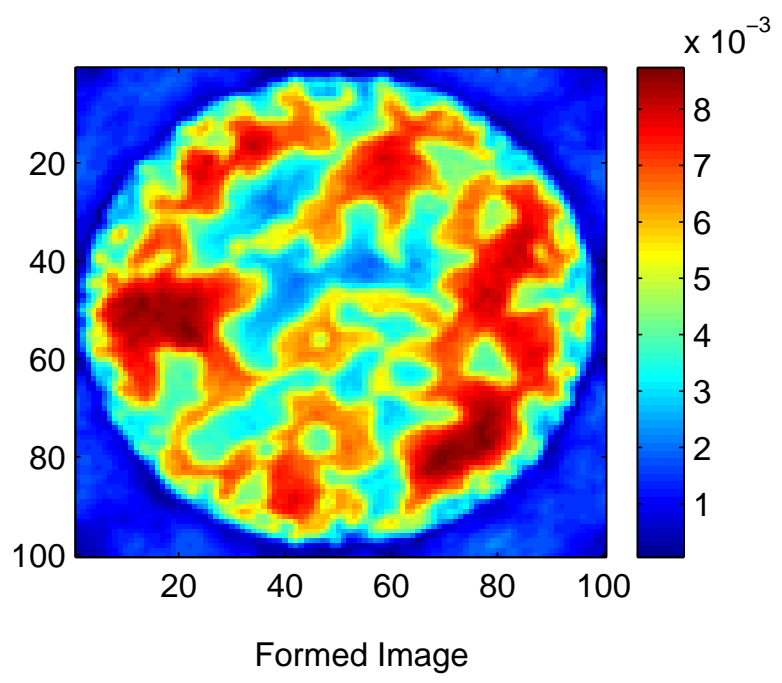



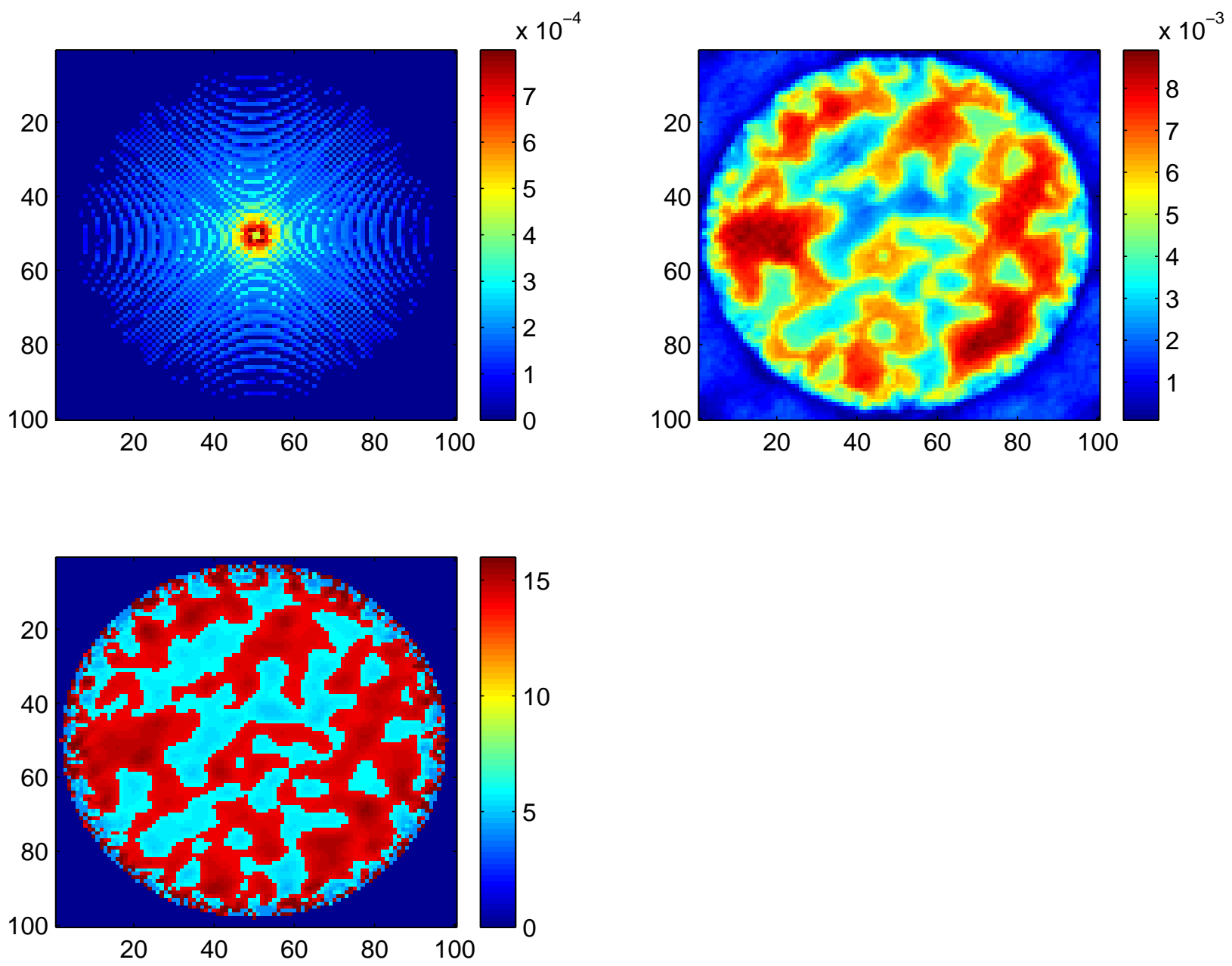

Figure 11. Image formed at $0.5 T_{f}$ by Trajectory 2 


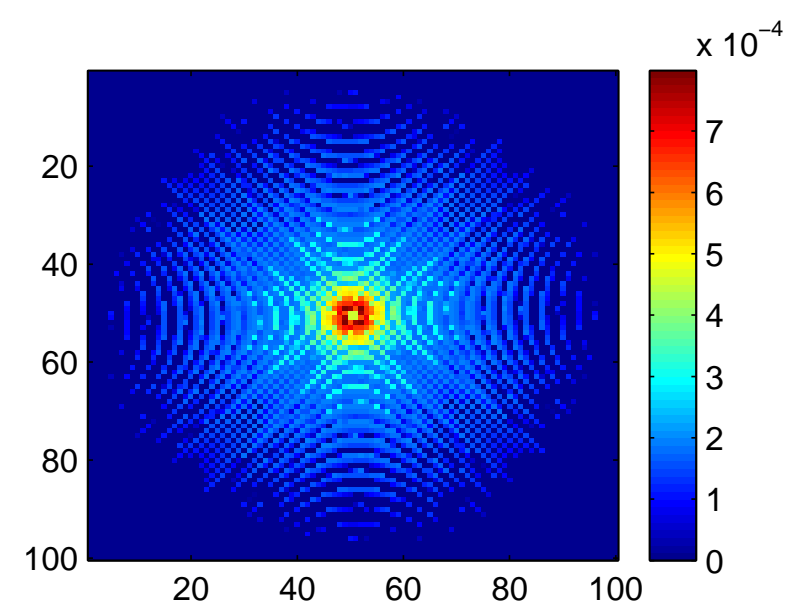

Spatial Frequency Coverage

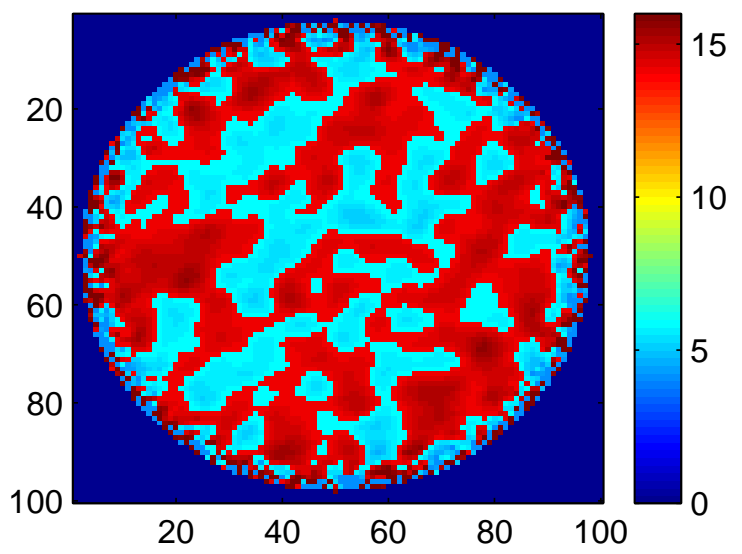

Actual Image

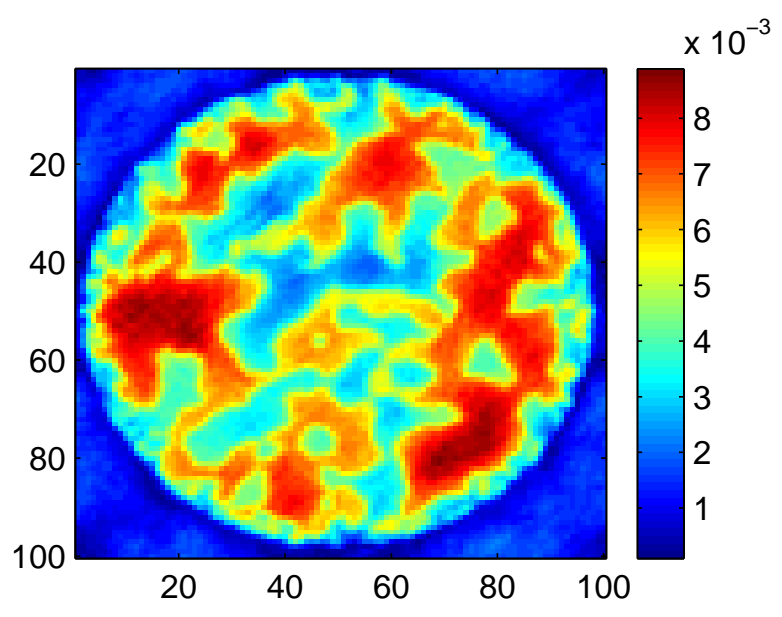

Formed Image

Figure 12. Image formed at $T_{f}$ by Trajectory 2 